\title{
Broadband absorption with gradient metasurfaces
}

\author{
Hoyeong Kwon, Hamidreza Chalabi, and Andrea Alù \\ Department of Electrical and Computer Engineering, The University of Texas at Austin, Austin, TX 78712, USA
}

Received: 29 September 2017 / Accepted: 28 November 2017

\begin{abstract}
A metasurface with appropriately designed transverse spatial inhomogeneities can provide the desired phase redistribution in response to an incident wave with arbitrary incident angle. This property of gradient metasurfaces has been used to modify light propagation in unusual manners, to transform the impinging optical wavefront with large flexibility. In this work, we show how gradient metasurfaces can be tailored to offer high absorption in thin absorptive layers, and how to design realistic metasurfaces for this purpose using dielectric materials.
\end{abstract}

\section{Introduction}

Metamaterials have attracted significant attention in recent years, for a wide range of applications, including invisibility, guiding phase or energy of electromagnetic waves in unusual manners, and several others [1-18]. The investigation of metasurfaces can be connected with a large body of work in the radio frequency spectrum studying the design and realization of frequency-selective surfaces and other thin resonant artificial screens for several decades [19-25]. With the development of nanotechnology and the possibility of fabricating nanoscale structures, similar methods have expanded towards the visible range of the electromagnetic spectrum [26-37]. Recent studies on metasurfaces have shown that it is possible to generalize the conventional Snell's law at the interface between two media, as well as controlling the power flow into different diffraction channels using periodic meta-gratings [38-40]. Metasurfaces are able to support these unusual reflection and refraction properties by leveraging spatial inhomogeneities over a surface that introduces phase variation on impinging wavefront, and reflect and refract in abnormal ways. In this paper, we envision that this approach, phase-gradient metasurfaces, can be realized under an absorptive layer, and tailored to introduce longer traveling paths to enhance energy harvesting and offer large absorption efficiency. Increasing the efficiency of solar energy harvesting can be particularly relevant, given the essentially unlimited amount of available energy. Using metasurfaces for solar energy

\footnotetext{
* e-mail: alu@mail.utexas.edu
}

harvesting applications can provide large efficiency enhancements, of direct importance for the growing field of nanophotonic management of solar panels.

Efficient light deflection and steering can be achieved by locally controlling the surface admittance and impedance over the surface $[27,28]$. The local reflection coefficient $\Gamma(x)$ and transmission $T(x)$ can be tailored to control locally the phase front, and imprinting on it a desired pattern [41,42]. The admittance distribution of ideal metasurfaces controls the phase of incident light over the complete $2 \pi$ phase range across the surface. Here, we assume normally incident light from the far-field, but, as shown in [34], the operation of gradient metasurfaces is inherently broadband and broad angle, so the response is expected to be robust to other incidence angles around normal direction, consistent with the major portion of impinging energy from the sun. Figure 1a shows how an ideal admittance profile can change the response of the surface under normal incident light. When normally incident light is deflected with metasurfaces towards an angle $\theta_{r}$, three main deflection modes are supported for deflection angles over $\theta_{r}>30^{\circ}$, and the other Floquet modes are evanescent. By locally tuning the surface impedance and admittance, we can make one diffraction order to be dominant compared to the other two, or we can scatter the incident light symmetrically into higher-order diffraction orders to be trapped inside the substrate.

Here, after studying the optimal impedance distributions to trap light into a small absorbing layer, we explore the realistic implementation of these metasurfaces using realistic nanostructured materials. We consider several requirements on the involved materials, including low-loss dielectric materials to avoid unwanted loss on the metasurface, and materials that can withstand moderately high temperatures, with optical properties robust against temperature variations. Considering these issues, $\mathrm{SiO}_{2}$ and 
a

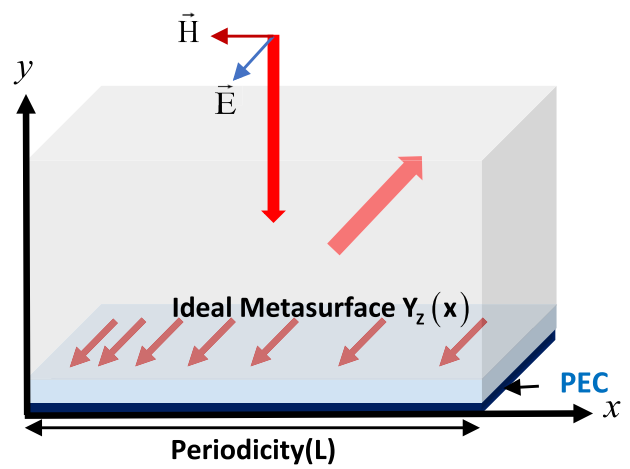

C

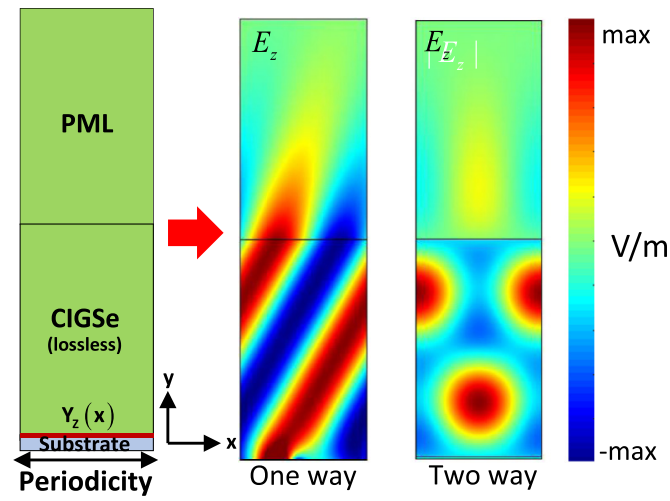

b

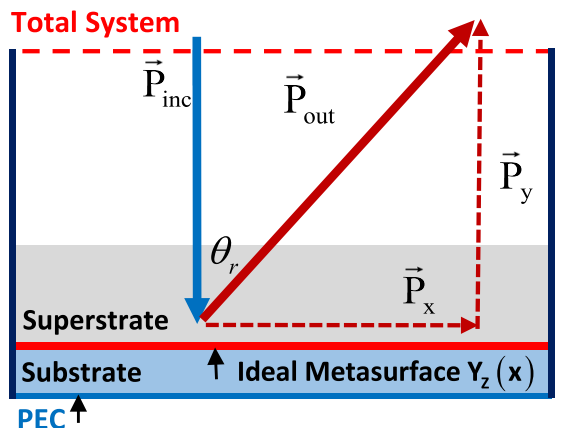

d
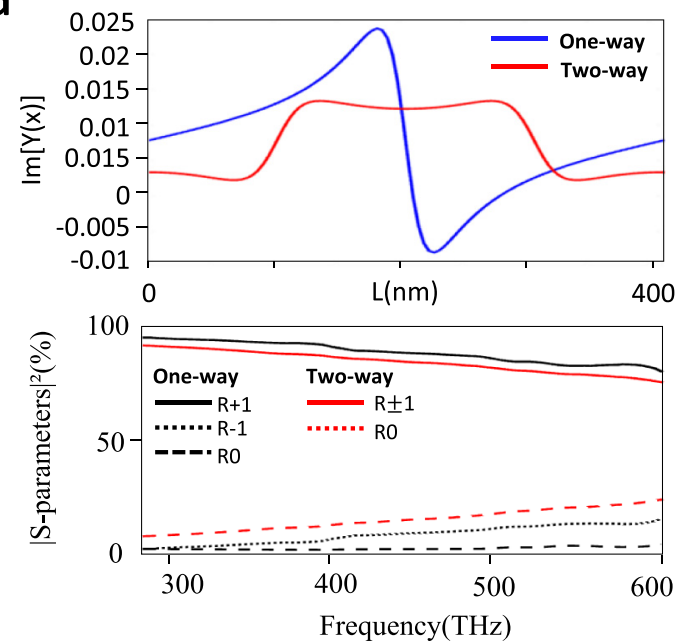

Fig. 1. (a) Schematic of the geometry under consideration. Light is shined from the normal direction with electric field $E_{z}$ and an admittance surface $Y_{z}(x)$ over a thin substrate and a ground plane. (b) Sketch of the relation between incident power $P_{\text {inc }}$ and deflected power $P_{\text {out }}$. When the metasurface is lossless, power is conserved in the system. (c) Geometry of interest to maximize deflection, assuming a lossless superstrate, i.e., ignoring the imaginary part of permittivity for the absorbing material. Scattered electric field profile for one-way and two-way deflection. For two-way deflection, the reflection phase is $\pi / 2$ for both beams. Frequency and deflection angle are set to $300 \mathrm{THz}$ and $60^{\circ}$,respectively (d) Admittance profile for one-way and two-way deflection, used in panel (c), and calculated reflection efficiency for both cases.

Si are good candidates for these applications. Both materials have high melting points and their permittivity is stable with temperature change [43,44]. Also, the permittivity gap between $\mathrm{SiO}_{2}$ and $\mathrm{Si}$ is relatively high, which provides good contrast to build dielectric metasurfaces [45]. For the absorptive layer, we consider CopperIndium-Gallium-Selenide (CIGSe). CIGSe is an $n$-type photovoltaic material that provides higher efficiency in light absorption compared to other $n$-type materials like $\mathrm{ZnO}$ or CdS $[46,47]$.

\section{Theoretical analysis}

The purpose of this work is to show how metasurfaces can be effective in increasing the absorption efficiency in thin layers. The geometry is shown in Figure 1a, and it corresponds to an ideal metasurface, backed by a substrate of thickness $d_{\text {subs }}$, assumed to be subwavelength, and a ground plane. When the wavelength in the absorptive material is $\lambda$ and the incidence and deflection angles of the metasurface are $\theta_{i}=0^{\circ}$ and $\theta_{r}$, respectively, the metasurface periodicity $L$ is given by $L=\lambda /\left|\sin \theta_{r}\right|$ at the frequency of interest. Once the periodicity is set for a specific deflection angle, the corresponding metasurface supports propagation channels in a discrete number of diffraction orders. For the calculation of admittances and deflection efficiency, we assume that the absorptive layer is lossless, discarding the imaginary part of the index. We also consider the case of surface admittance $\overline{\bar{Y}}$ to be a scalar $Y_{z}(x)$, which corresponds to the case of isotropic surfaces, or of absence of polarization coupling under an incident electric field $\vec{E}=E_{0} \hat{z}$ and impinging from the $y$ direction. The desired wave number of the deflected beam is therefore $\vec{k}_{r}=k_{u p}\left(\cos \theta_{r} \hat{y} \pm \sin \theta_{r} \hat{x}\right)$, where $k_{u p}=$ $\omega R e[\sqrt{\varepsilon \mu}]=k_{0} \operatorname{Re}\left[\sqrt{\varepsilon_{u p}}\right]$ is the propagation constant in the superstrate layer, and \pm signs refer to the direction of the deflected beams in the transverse plane. The required admittance to deflect light towards one of the two anomalous reflection channels can be derived based on the 
a

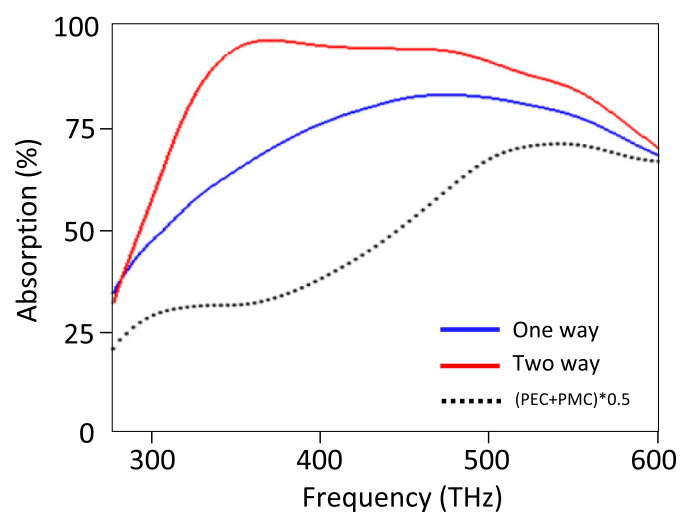

C
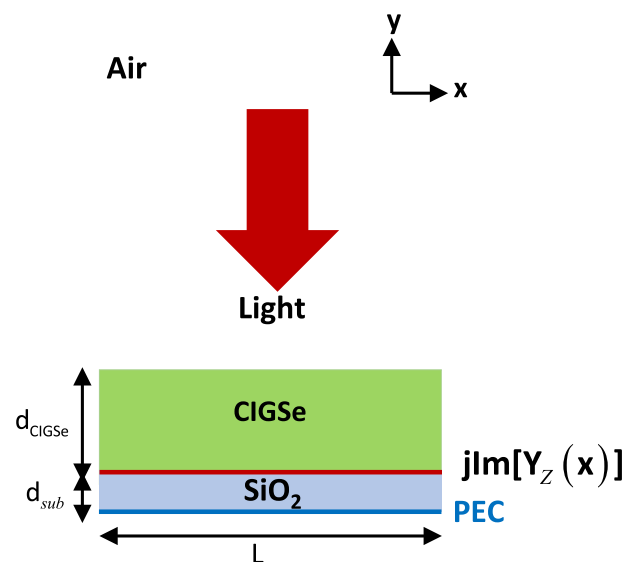

b

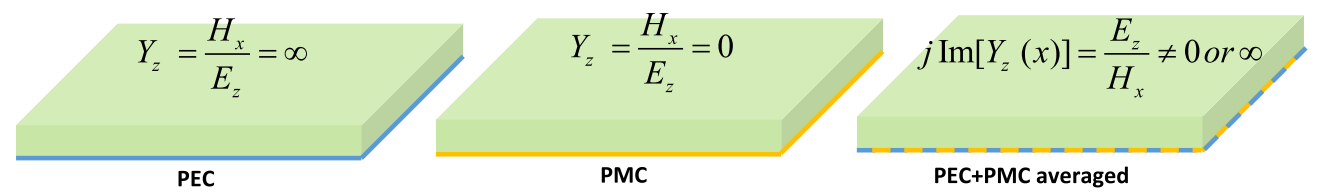

Fig. 2. (a) Absorption efficiency when ideal metasurfaces are used beneath the substrate compared to the case of simple reflector backing. (b) Relationship between PEC and PMC boundary conditions and admittance. (c) Schematic of the realistic geometry for our simulations, $d_{\text {cigse }}$ is $100 \mathrm{~nm}$ and $d_{\text {subs }}$ is $50 \mathrm{~nm}$. Periodicity L and $j \operatorname{Im}\left[Y_{z}(x)\right]$ are changed for each deflection angle and frequency.

analysis in [41,42]. With incident angle $\theta_{i}=0^{\circ}$, the admittance profile is

$$
\begin{aligned}
Y_{z}^{1}(x) & =\frac{\sqrt{\varepsilon_{u p}}}{\eta_{0}} \frac{\left(1-\Gamma_{o} \cos \theta_{r} e^{-j k_{u p} \sin \theta_{r} x}\right)}{\left(1+\Gamma_{o} e^{-j k_{u p} \sin \theta_{r} x}\right)} \\
& +\frac{j \sqrt{\varepsilon_{\text {subs }}}}{\eta_{0}} \cot \left(k_{\text {subs }} d_{\text {subs }}\right) .
\end{aligned}
$$

If instead we want to deflect light towards two anomalous reflection channels with reflection coefficients $\Gamma_{t 1}, \Gamma_{t 2}$, the required admittance profile becomes

$$
\begin{aligned}
Y_{z}^{2}(x) & =\frac{\sqrt{\varepsilon_{u p}}}{\eta_{0}} \frac{\left(1-\cos \theta_{r}\left(\Gamma_{t 1} e^{-j k_{u p} \sin \theta_{r} x}+\Gamma_{t 2} e^{j k_{u p} \sin \theta_{r} x}\right)\right)}{\left(1+\Gamma_{t 1} e^{-j k_{u p} \sin \theta_{r} x}+\Gamma_{t 2} e^{k_{u p} \sin \theta_{r} x}\right)} \\
& +\frac{j \sqrt{\varepsilon_{s u b s}}}{\eta_{0}} \cot \left(k_{\text {subs }} d_{\text {subs }}\right) .
\end{aligned}
$$

The imaginary part in both expressions compensates the phase delay introduced by the substrate. $k_{\text {subs }}$ is the wave number in the substrate and $\varepsilon_{\text {subs }}$ is the corresponding permittivity. Power conservation imposes some constraints on the amplitude of the reflection coefficients to ensure a globally lossless metasurface, as sketched in Figure 1b. In particular, for a metasurface that does not globally provide energy to the impinging wave, the tilt to an oblique angle from normal incidence implies that there is an increase in power density, proportional to $1 / \cos \theta_{r}$ for a single deflection angle. As noticed in [41], even for a globally lossless metasurface, equation (1) implies the need of a continuous combination of loss and gain across the surface to ensure unitary efficiency, and this need can be mitigated with the use of more advanced metasurface designs [39]. The admittance profile for deflection towards two channels, as in equation (2), has similar challenges.

Figure 1c shows the scattered E-field when the admittance is designed to deflect the incident impinging energy towards $60^{\circ}$ propagating into one and two directions, respectively for the simulation panel on the left and on the right. The field profile for one-way deflection clearly shows that the beam is deflected towards $60^{\circ}$, and two ways deflection shows the interference of waves propagating in opposite directions. Energy conservation is achieved by choosing the proper amplitude of $\Gamma$, but the need for active/passive impedance distributions to ensure $100 \%$ efficiency in deflection is hard to realize in various applications. For this reason, in the following we design the surfaces using only the imaginary part of the required admittance profile, and discarding the real part, as suggested in [41]. This approximation is accurate as long as the deflection angle is not extreme.

The phase relationship between the two reflected beams in two-way deflection mode can be adjusted at will from 0 to $2 \pi$. When both reflection coefficients have the same phase of $\pi / 2$, as considered in Figure 1c, right panel, the required impedance profile ensures minimized real part, therefore enabling the strongest coupling efficiency using a fully passive metasurface. For this reason, in the following, we choose this specific configuration for the two-deflection metasurface. The top panel in Figure 1d shows the values of $\operatorname{Im}\left[Y_{z}^{1}(x)\right]$ and $\operatorname{Im}\left[Y_{z}^{2}(x)\right]$ extracted from equation (1) and (2) for $\theta_{r}=60^{\circ}$ at $300 \mathrm{THz}$. In the bottom panel, we plot the calculated reflection coefficient towards the anomalous direction in the two cases of one-way and two-way deflection over a broad range of frequencies, beyond the 
a

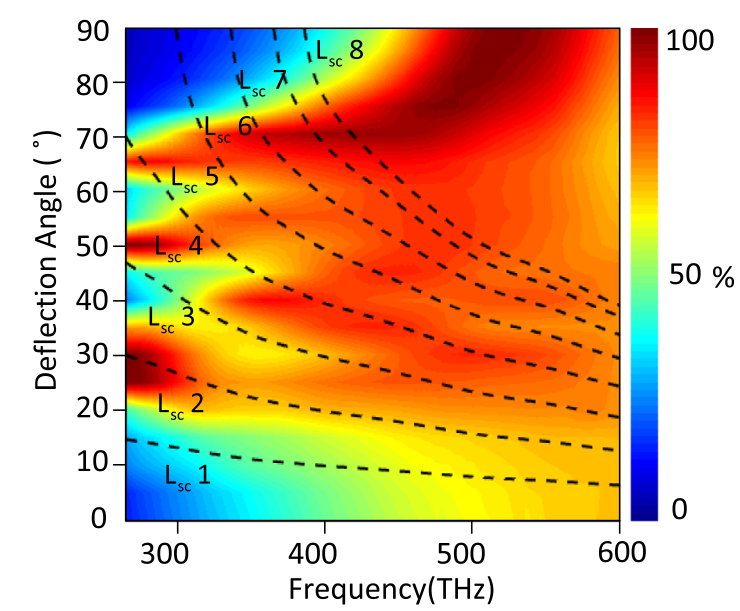

C

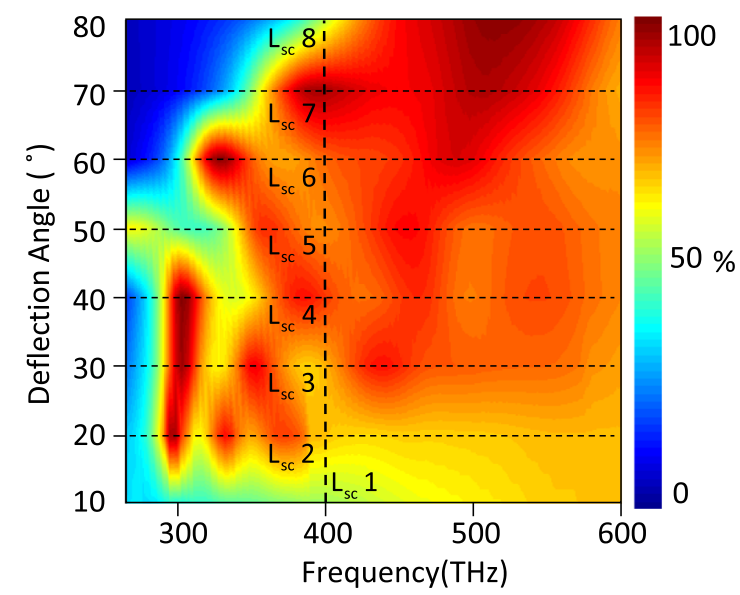

b

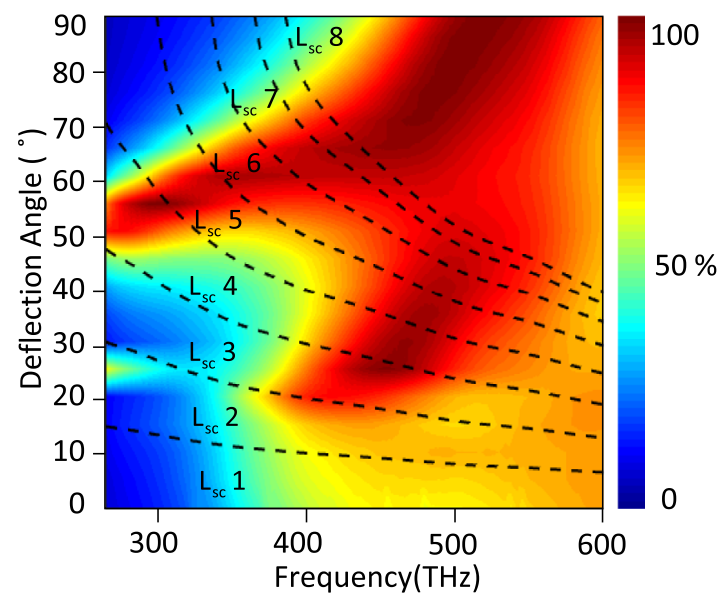

d

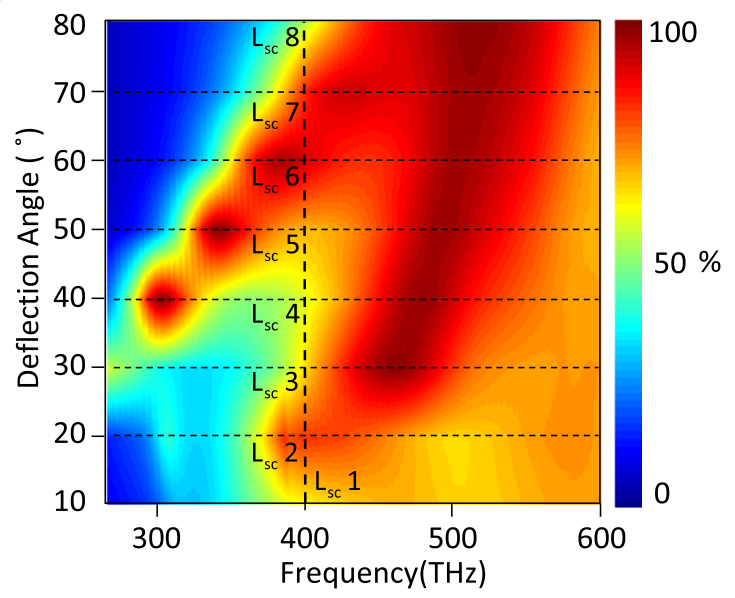

Fig. 3. (a) and (b) show the absorption efficiency for $\operatorname{Im}\left[Y_{z}(x)\right]$ beneath a thin CIGSe layer. The black dashed lines consider the natural angular dispersion with frequency for a fixed period, and the labels correspond to $400 \mathrm{THz}$, with deflection angles from $10^{\circ}$ to $80^{\circ}$. (c) and (d) show the same results for metasurfaces wtih fixed periodicity.

design frequency. Due to the finite substrate thickness, the reflection efficiency drops slowly when frequency is increased, with the residual power being channeled towards normal incidence. This panel clearly shows the broadband nature of the gradient metasurface approach, which is a significant advantage for solar energy [34,42].

We now add the loss in the superstrate layer, and explore ways to use the wave deflection to enable broadband absorption. Figure 2a shows the absorption efficiency for the admittance profiles in Figure 1d. The red solid line corresponds to two-way deflection and the blue solid line to one-way deflection. Both $j \operatorname{Im}\left[Y_{z}^{1}(x)\right]$ and $j \operatorname{Im}\left[Y_{z}^{2}(x)\right]$ achieve high absorption compared to the case with no metasurface, over a broad range of angles. In the plot, we compare the absorption to the case in which the absorptive substrate is backed by a perfect electric conductor (PEC) and by a perfect magnetic conductor (PMC), and average the two. The reason for this specific choice is that the boundary condition in equation (1) and (2) imposes a phase of the reflection coefficient in the transverse direction that spans from 0 to $\infty$, i.e., it gradually varies from the phase of a PMC and a PEC boundary, as sketched in Figure 2b.

For realistic metasurfaces, we need to consider the discretization of the ideal admittance profile. In addition, field coupling between the various sub-sections plays an important role. This problem can be handled by adjusting the difference in permittivity between the considered materials [31]. The index of the superstrate also affects the efficiency of metasurfaces. With a high index layer, the phase varies over a short distance, complicating the design. In this work, $\mathrm{Si}$ and $\mathrm{SiO}_{2}$ are used to realize the metasurface, and CIGSe is the absorbing superstrate. The permittivity of CIGSe is around 8 and permittivity of $\mathrm{Si}$ and $\mathrm{SiO}_{2}$ is approximately 12 and 2, respectively, which is a sufficient contrast to realize efficient two-way deflection, but it does not provide good performance for the case of one-way deflection, as discussed in the following section. 
a

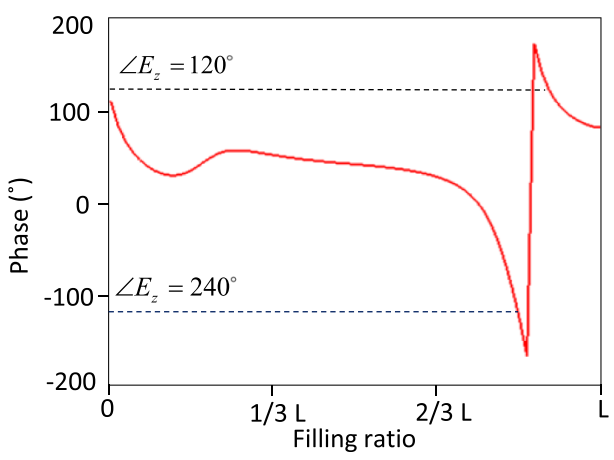

b

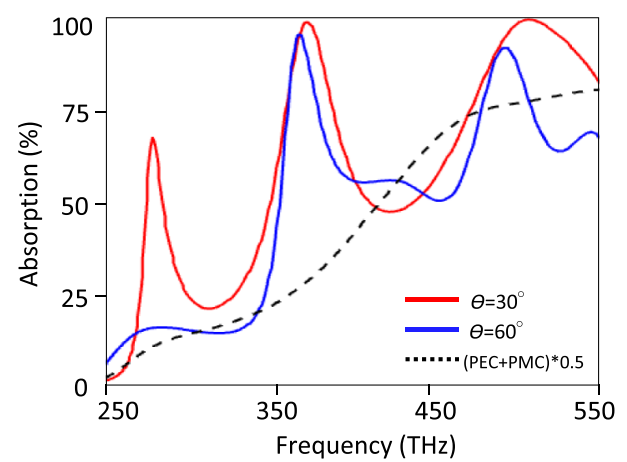

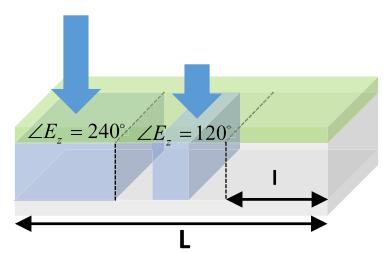

One-way

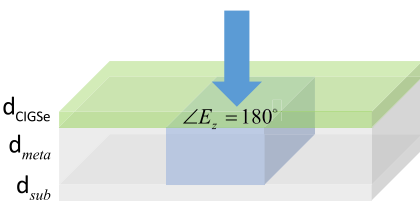

Two-way
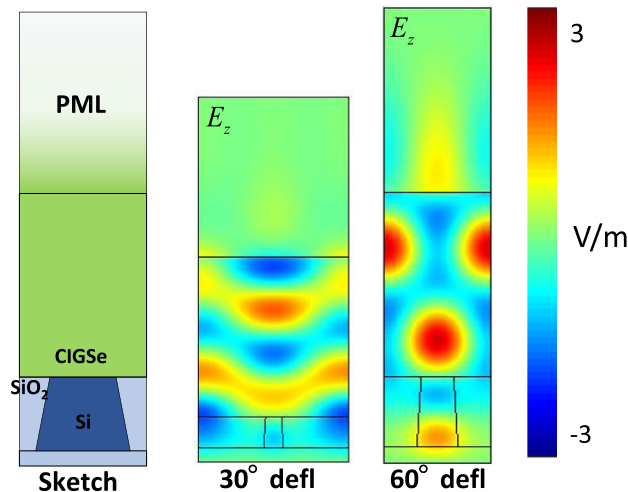

Fig. 4. (a) Phase profile when nanoparticle width is sweeped inside one periodicity for a three-block discretized metasurface. Si and $\mathrm{SiO}_{2}$ are used for making the metasurface. Sketch in the right shows the geometry of the discretized metasurfaces. (b) When the frequency is set at $400 \mathrm{THz}$, absorption has a similar value as in the case of ideal metasurfaces. Both cases are better than in the case with no metasurface. The right panel shows the scattered E-field profiles, confirming large light trapping.

\section{Results}

\subsection{Ideal metasurfaces}

We start considering the case of ideal metasurface profiles $j \operatorname{Im}\left[Y_{z}(x)\right]$ applied on top of a $\mathrm{SiO}_{2}$ substrate. We consider deflection angles from $0^{\circ}$ to $90^{\circ}$ in steps of $5^{\circ}$, and a frequency range from $280 \mathrm{THz}$ to $600 \mathrm{THz}$ for the operation frequency. The sketch of the geometry in our simulation is shown in Figure 2c. The thickness of the absorbing CIGSe superstrate is $100 \mathrm{~nm}$. We show in Figure $3 \mathrm{a}$ and $\mathrm{b}$ the total absorption for one-way, and two-way metasurfaces. For two-way metasurfaces, phases of both beams are set to $0.5 \pi$ making the relative phase of 0 and the sum of $\pi$, which maximizes the effect of imaginary part as mentioned above. Absorption efficiency tends to increase with frequency, as expected, because of the increased electrical thickness of the CIGSe layer. Absorption increases with the deflected angle, and an absorption peak is observed around $60^{\circ}$ to $70^{\circ}$. Critical angle at the CIGSe to air interface is around $70^{\circ}$ in the entire frequency range considered here, so that the total reflection contributes to increase light trapping, and boosts the absorption efficiency to close to $100 \%$ over a broad bandwidth and angular range. Given that the decay constant in CIGSe at $400 \mathrm{THz}$ is $\alpha=-2.03 \times 10^{6} / \mathrm{m}$, we calculate that $35 \%$ of energy is absorbed over one pass in $100 \mathrm{~nm}$ thickness. Overall area of 2D color map shows high absorption, but there are several points where absorption is low, especially in the left side of the plots, for which the electrical length of the superstrate is shorter, and therefore the optimal choice of deflection angle is more important.

The absorption efficiency in Figure $3 \mathrm{a}$ and $\mathrm{b}$ is calculated assuming that the admittance profiles are designed optimally for each deflection angle and frequency. In practice, once a metasurface is designed and realized, the periodicity is fixed by the design frequency and angle, and there is a natural dispersion of anomalous reflection at different frequencies. We consider this effect by plotting black dashed lines in Figure $3 \mathrm{a}$ and $\mathrm{b}$, which show the dispersion of deflection angle with frequency, and may be considered realistic regions over which broadband absorption can be achieved with a fixed metasurface period.

Figure 3c, d show the same data following the dashed lines. In this case, the deflection angle in the vertical axis is defined based on designs at $300 \mathrm{THz}$. In these plots, we can see more clearly the optimal choice of metasurface periodicity to enable broadband absorption. High absorption can be clearly achieved at specific periodicity values. 


\subsection{Realistic metasurfaces}

Several approaches have been studied to implement realistic metasurfaces that can tailor the impinging beams at will. In this study, phase-gradient metasurfaces are considered, due to their inherent broad bandwidth and design robustness. Since the deflection angle required for light trapping is around $60^{\circ}-70^{\circ}$, based on the results in the previous section, the deflection efficiency even in the case of totally passive gradient metasurfaces is expected to be rather high. After some optimizations, we have found that discretization with three unit-cells per period is a reasonable trade-off between fabrication complexity and absorption efficiency. Figure 4a shows the designed metasurfaces for both one-way and two-way deflections (top and bottom, respectively), using $\mathrm{Si}$ and $\mathrm{SiO}_{2}$ metasurfaces. By sweeping the width of $\mathrm{Si}$ blocks in $\mathrm{SiO}_{2}$, we can derive the relative phase of the local reflection coefficient, which synthesizes the required discretized admittance profile. In these results, we assumed again $d_{\text {meta }}=100 \mathrm{~nm}$ and $d_{\text {sub }}=50 \mathrm{~nm}$ at $400 \mathrm{THz}$. From this plot, it is clear that, for the given materials, it is not possible to realize a complete $360^{\circ}$ phase span, which makes it particularly challenging to realize one-way deflection metasurfaces. However, metasurfaces for two-way deflection can be fabricated since due to symmetry, they can be realized using a single Si block per period.

Figure $4 \mathrm{~b}$ shows the calculated absorption for optimized realistic metasurfaces over the CIGSe layer. After discretization, we have found some small deviations on the optimal deflection angles compared to the ideal metasurface scenario, and we show here the case of $30^{\circ}$ and $60^{\circ}$, which correspond to optimal choices for two-way discretized deflection metasurfaces, as seen in Figure 3d. The simulations confirm that it is possible to achieve good broadband absorption with a peak efficiency over $95 \%$, and a significant improvement compared to the case with no metasurfaces. Field profiles for each deflection angle at the design frequency of $400 \mathrm{THz}$ are shown in the insets of Figure $4 \mathrm{~b}$, confirming the deflection efficiency and large light trapping. These symmetric metasurfaces for two-way deflection may appear consistent with other approaches for nanophotonic management of solar cells, using Mie resonant structures [47-50]. However, here we consider non-resonant blocks, geared at manipulating the local phase of the reflection coefficient for broadband light trapping, and purposefully avoiding single resonant elements associated to narrower bandwidths of operation.

\section{Conclusions}

Metasurfaces can offer interesting opportunities for enhanced absorption in thin layer materials. These may be of interest for thin-film solar cell applications, as well as for light emission enhancement in LEDs. In this paper, we first derived the optimal performance for light deflection and trapping assuming ideal admittance profiles, and then we applied these results to realistic dielectric metasurfaces. Our results show that high value of absorption efficiency may be achieved considering both one-way and two-way deflection metasurfaces, but given the constraints on fabrication in the visible range, two-way metasurfaces may provide an easier path to the practical implementation of these structures. We believe that these broadband nanophotonic solutions to absorption enhancement may have an impact in various applications of current interest, including photovoltaics and light emission.

This work was supported in part by the Air Force Office of Scientific Research and the Welch Foundation with grant No. F1802. We acknowledge useful discussions with V. Neder and A. Polman.

\section{References}

1. J. Li, J.B. Pendry, Hiding under the carpet: A new strategy for cloaking, Phys. Rev. Lett. 101, 203901 (2008)

2. D.R. Smith, W.J. Padilla, D. Vier, S.C. Nemat-Nasser, S. Schultz, Composite medium with simultaneously negative permeability and permittivity, Phys. Rev. Lett. 84, 4184 (2000)

3. J.B. Pendry, Negative refraction makes a perfect lens, Phys. Rev. Lett. 85, 3966 (2000)

4. C.M. Soukoulis, M. Wegener, Past achievements and future challenges in the development of three-dimensional photonic metamaterials, Nat. Photonics. 5, 523-530 (2011)

5. A. Alù, N. Engheta, Multifrequency optical invisibility cloak with layered plasmonic shells, Phys. Rev. Lett. 100, 113901 (2008)

6. A. Alù, N. Engheta, Achieving transparency with plasmonic and metamaterial coatings, Phys. Rev. E. 72, 016623 (2005)

7. A. Alù, Mantle cloak: invisibility induced by a surface, Phys. Rev. B. 80, 245115 (2009)

8. C. Argyropoulos, P.Y. Chen, G. D'Aguanno, N. Engheta, A. Alù, Boosting optical nonlinearities in $\varepsilon$-near-zero plasmonic channels, Phys. Rev. B. 85, 045129 (2012)

9. J.D. Joannopoulos, S.G. Johnson, J.N. Winn, R.D. Meade, Photonic crystals: molding the flow of light (Princeton University Press, Princeton, NJ 2008)

10. R.W. Ziolkowski, E. Heyman, Wave propagation in media having negative permittivity and permeability, Phys. Rev. E. 64, 056625 (2001)

11. W.M. Zhu, Q.H. Song et al., A random access reconfigurable metamaterial and a tunable flat lens, Adv. Mat. 271, 47394743 (2015)

12. U. Leonhardt, Optical conformal mapping, Science $\mathbf{3 1 2}$, 1777-1780 (2006)

13. U. Leonhardt, T. Tyc, Broadband invisibility by noneuclidean cloaking, Science 323, 110-112 (2009)

14. R. Liu, C. Ji, J.J. Mock, J.Y. Chin, T.J. Cui, D.R. Smith, Broadband ground-plane cloak, Science 323, 366-369 (2009)

15. E. Kallos, C. Argyropoulos, Y. Hao, Ground-plane quasi cloaking for free space, Phys. Rev. A. 79, 063825 (2009)

16. L. Novotny, N. van Hulst, Antennas for light, Nat. Photonics. 5, 83-90 (2011)

17. Q. Wu, T. D. Hewitt, X.-C. Zhang, Two-dimensional electrooptic imaging of THz beams, Appl. Phys. Lett. 69, 1026 (1996)

18. L. Huang, X. Chen, H. Mühlenbernd, H. Zhang, S. Chen, B. Bai, Q. Tan, G. Jin, K.-W. Cheah, C.-W. Qiu, J. Li, T. Zentgraf, S. Zhang, Three-dimensional optical holography using a plasmonic metasurface, Nat. Commun. 4, 2808 (2013) 
19. H. Steyskal, A. Hessel, J. Shmoys, On the gain-versus-scan trade-offs and the phase gradient synthesis for a cylindrical dome antenna, IEEE Antennas Propag. 27, 825-831 (1979)

20. M. Navarro-Cía, M. Aznabet, M. Beruete, F. Falcone, O. El Mrabet, M. Sorolla, M. Essaaidi, Stacked complementary metasurfaces for ultraslow microwave metamaterials, Appl. Phys. Lett. 96, 164103 (2010)

21. J. Romeu, Y. Rahmat-Samii, Fractal FSS: a novel dual-band frequency selective surface, IEEE Antennas Propag. 48, 1097-1105 (2000)

22. K. Sarabandi, N. Behdad, A frequency selective surface with miniaturized elements, IEEE Trans. Antennas Propag. 55, 1239-1245 (2007)

23. A. Ben Munk, Frequency selective surfaces: theory and design (John Wiley, New York, 2000)

24. A. Abbaspour-Tamijani, K. Sarabandi, G.M. Rebeiz, Antenna-Filter-Antenna arrays as a class of bandpass frequency-selective surfaces, IEEE Trans. Microw. Theory Tech. 52, 1781-1789 (2004)

25. R. Mittra, C. H. Chan, T. Cwik, Techniques for analyzing frequency selective surfaces-a review, IEEE 76, 1593-1615 (1988)

26. N. Yu, P. Genevet, M.A. Kats, F. Aieta, J.-P. Tetienne, F. Capasso, Z. Gaburro, Light propagation with phase discontinuities: generalized laws of reflection and refraction, Science 334, 333-337 (2011)

27. C.L. Holloway, M.A. Mohamed, E.F. Kuester, A. Dienstfrey, Reflection and transmission properties of a metafilm: with an application to a controllable surface composed of resonant particles, IEEE Electromagn. Compat. 47, 853-865 (2005)

28. C.L. Holloway, E.F. Kuester, J.A. Gordon, J. O'Hara, J. Booth, D.R. Smith, An overview of the theory and applications of metasurfaces: the two-dimensional equivalents of metamaterials, IEEE Antennas Propag. 54, 10-35 (2012)

29. C. Pfeiffer, A. Grbic, Bianisotropic metasurfaces: ultra-thin surfaces for complete control of electromagnetic wavefronts, Phys. Rev. Applied. 2, 044011 (2014)

30. F. Monticone, N.M. Estakhri, A. Alù, Full control of nanoscale optical transmission with a composite metascreen, Phys. Rev. Lett. 110, 203903 (2013)

31. N.M. Estakhri, A. Alù, Ultra-thin unidirectional carpet cloak and wavefront reconstruction with graded metasurfaces, IEEE Antennas Propag. 13, 1775-1778 (2014)

32. N.M. Estakhri, A. Alù, Recent progress in gradient metasurfaces, J. Opt. Soc. Am. B. 33, A21 (2015)

33. N.M. Estakhri, V. Neder, M.W. Knight, A. Polman, A. Alù, Visible light, wide-angle graded metasurface for back reflection, ACS Photonics. 4, 228-235 (2017)
34. N. Mohammadi Estakhri, A. Alù, Manipulating optical reflections using engineered nanoscale metasurfaces, Phys. Rev. B. 89, 235419 (2014)

35. A. Fallahi, J. Perruisseau-Carrier, Design of tunable biperiodic graphene metasurfaces, Phys. Rev. B. 86, 195408 (2012)

36. X. Ding, F. Monticone, K. Zhang, L. Zhang, D. Gao, S.N. Burokur, A. de Lustrac, Q. Wu, C.-W. Qiu, A. Alù, Ultrathin pancharatnam-berry metasurface with maximal cross-polarization efficiency, Adv. Material. 27, 1195-1200 (2015)

37. Y. Zhao, Manipulating light polarization with ultrathin plasmonic metasurfaces, Phys. Rev. B. 84, 205428 (2011)

38. C. Pfeiffer, A. Grbic, Metamaterial Huygens' surfaces: tailoring wave fronts with reflectionless sheets, Phys. Rev. Lett. 110, 197401 (2013)

39. Y. Ra'di, D.L. Sounas, A. Alù, Metagratings: beyond the limits of graded metasurfaces for wave front control, Phys. Rev. Lett. 119, 067404 (2017)

40. H. Chalabi, Y. Ra 'di, D.L. Sounas, A. Alù, Efficient anomalous reflection through near-field interactions in metasurfaces, Phys. Rev. B. 96, 075432 (2017)

41. N. Mohammadi Estakhri, A. Alù, Wave-front transformation with gradient metasurfaces, Phys. Rev. X. 6, 041008 (2016)

42. A. Alù, N.M. Estakhri, C. Argyropoulos, Graded metascreens to enable a new degree of nanoscale light management, Phil. Trans. R. Soc. A. 373, 20140351 (2015)

43. D.B. Leviton, B.J. Frey, Temperature-dependent absolute refractive index measurements of synthetic fused silica, Conference. Proc. of SPIE, 6273, 62732K (2006)

44. D.B. Leviton, B. J. Frey, Temperature-dependent refractive index of silicon and germanium, Conference. Proc. of SPIE, 6273, 62732J (2006)

45. E.D. Palik, Handbook of optical constants of solids (Academic Press, San diego 1998)

46. J. Hedstrom, H. Ohlsen, M. Bodegard, A. Kylner, L. Stolt, D. Hariskos, M. Ruckh, H.-W. Schock, ZnO/CdS/Cu(InGa) $\mathrm{Se} / \mathrm{sub} 2 /$ thin film solar cells with improved performance, IEEE Photovoltaic Specialists Conference, 364 (1993)

47. C. Van Lare, G. Yin, A. Polman, M. Schmid, Light coupling and trapping in ultrathin $\mathrm{Cu}(\mathrm{In}, \mathrm{Ga}) \mathrm{Se} 2$ solar cells using dielectric scattering patterns, ACS Nano. 9, 9603-9613 (2015)

48. M. Schmid, G. Yin, M. Song, S. Duan, B. Heidmann, D. Sancho-Martinez, S. Kämmer, T. Köhler, P. Manley, Mc. Lux-Steiner, Concentrating light in $\mathrm{Cu}(\mathrm{In}, \mathrm{Ga}) \mathrm{Se} 2$ solar cells, Proc. SPIE, 9937, 993703 (2016)

49. Y. Tak, S. Joon Hong, J. Sung Lee, K. Yong, Fabrication of $\mathrm{ZnO} / \mathrm{CdS}$ core/shell nanowire arrays for efficient solar energy conversion, J. Mat. Chem. 19, 5945-5951 (2009)

50. G. Yin, A. Steigert, P. Andrae, M. Goebelt, M. Latzel, P. Manley, I. Lauermann, S. Christiansen, M. Schmid, Integration of plasmonic $\mathrm{Ag}$ nanoparticles as a back reflector in ultra-thin $\mathrm{Cu}(\mathrm{In}, \mathrm{Ga}) \mathrm{Se} 2$ solar cells, Appl. Surf. Sci. 355, 800-804 (2015)

Cite this article as: Hoyeong Kwon, Hamidreza Chalabi, Andrea Alù, Broadband absorption with gradient metasurfaces, EPJ Appl. Metamat. 2018, 5, 4 